DOE / PC90308-8

\title{
A NEW MODEL OF COAL-WATER INTERACTION AND RELEVANCE FOR DEWATERING
}

E. M. SUUBERG (PRINCIPAL INVESTIGATOR)

Y. YUN, W.D. LILLY (STAFF)

K. LEUNG AND TOM GATES (STUDENTS)

DIVISION OF ENGINEERING

BROWN UNIVERSITY

PROVIDENCE, RI 02912

TEL. (401) 863-1420

\section{QUARTERLY TECHNICAL PROGRESS REPORT 1 JUNE- 31 AUGUST, 1992}

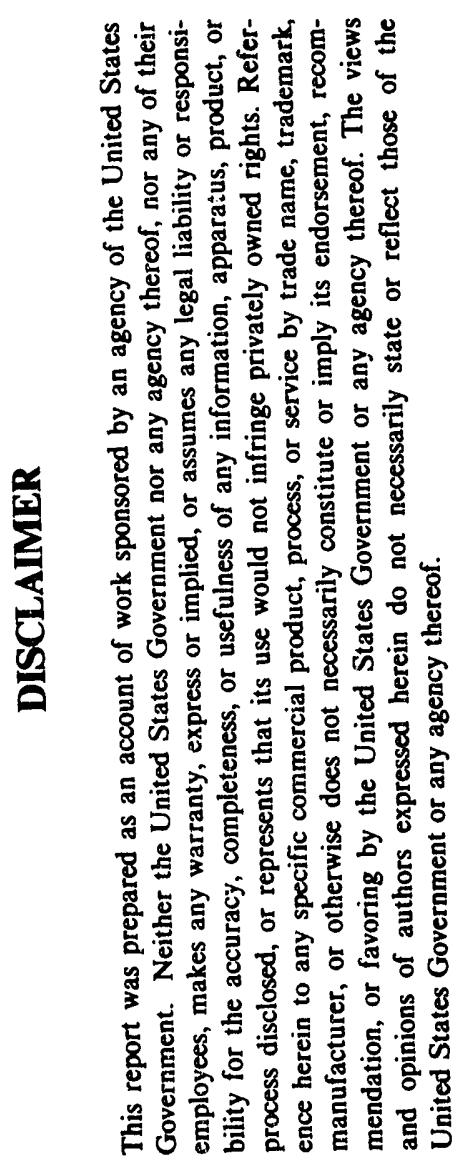

PREPARED FOR:

U. S. DEPT. OF ENERGY

PITTSBURGH ENERGY TECHNOLOGY CENTER

P.O. BOX 10940

PITTSBURGH, PA 15236

DR. WU-WEY WEN

TECHNICAL PROJECT OFFICER

"US/DOE Patent Clearance is not required prior to the publication of this document" 


\section{Project Overview}

This project is concerned with a basic scientific question concerning the properties of coal - to what extent is the ability of coal to hold moisture a manifestation of the well-known ability of coal to swell, when exposed to good solvents? The question implies that the long-held belief that coal holds a significant portion of its moisture by classical capillary condensation processes, is possibly in error. This seems to be a very real possibility for low rank coals- i.e. lignites. To explore this hypothesis further requires an examination of the basic phenomena governing the swelling of coals in good solvents. This is the focus of the first part of this project.

The possibility that coal holds a significant portion of its moisture by solvent swelling mechanisms leads to an interesting technical issue. It is well known that simple drying of low rank coals at minemouth is ineffective because the process is reversible, to a significant degree. The economic advantages of pre-shipment drying have however dictated a search for "permanent" drying procedures. These have been developed by largely empirical means, and involve mild pyrolytic treatments of the coals in oil, steam or liquid water itself. The idea has always been to pyrolytically remove oxygen groups, which are assumed to be those that hold water most strongly by hydrogen bonding. The treatments have been designed to minimize tar formation and decrepitation of the particles, both highly undesirable. In relation to the present new hypothesis concerning water retention, it is likely that a sound approach to permanent drying would involve highly crosslinking the coal at mild drying conditions. The crosslinked coal could not swell sufficiently to hold much water. It is identifying processes to achieve this goal, that constitute the objective of the second phase of this work.

\section{Current Ouarter Summary}

This quarter's efforts involved beginning to regularize the use of the dynamic mechanical analysis (DMA) technique to obtain structural information about coals. The information feeds directly into the development of a more realistic model of coal-solvent (water) interactions, since as 
has been earlier noted, it is necessary to measure separately the elastic constants of coals, and the mixing enthalpies. This report focuses on the elastic changes that coal undergoes in response to thermal treatment of the coal (as may be of relevance to the drying of coal), and exposure of the coal to solvents.

\section{Next Ouarter Plans}

We need to develop further the theory of coal swelling with dissociation that was developed and reported in a previous quarterly report. The model has not worked well with respect to predicting the behavior of water, perhaps because the mixing enthalpy effects are not yet well represented for that case, or perhaps because the elastic nature of coal structure is not yet well represented.

Exploration of the elastic response of coals will continue. There has been identified a problem with the equipment we are currently using, in that the calibration wanders. We are forced to send the equipment in for service. The nature of the problem will be discussed below in connection with the results presented here. 


\section{INTRODUCTION}

It is now well established that thermal pretreatment in a range of temperatures lower than those needed for pyrolysis can significantly affect the macromolecular structure of coals. These effects are of concern here, where the role of water in the structure of coal, and also the effects of heating to remove this water, are of interest.

The present study is concerned with the effects of heat/solvent exposures on the elastic constants of the coal network structure. This, in turn, tells one about how the non-covalent interactions in coal are being broken down. Studies of the elastic properties of coal are nothing new [e.g. 1-10]. Because of the compressibility of coal, the application of mercury porosimetry to pore characterization in coal has involved careful corrections, so that the actual pore size distribution could be calculated $[1,11,12]$. However, the limitation caused by compressibility of bulk coaf structure can be an advantage in revealing the structural changes during drying processes and in establishing a suitable elastic model for macromolecular coal structure.

It is known that dynamic mechanical methods are ca. 1000 times more sensitive for detecting molecular relaxations such as the glass transition temperature, $T_{\mathbf{g}}$, than are differential scanning calorimetry (DSC)/differential thermal analysis (DTA) techniques [13]. Weller and Wert [7-10] have extensively applied a torsion pendulum technique, one of the dynamic mechanical methods, for elucidating the elastic properties of coal in the temperature range below $200^{\circ} \mathrm{C}$. They employed square rods cut from whole coal. Here we seek to examine the thermal structural relaxation as a function of temperature by employing dynamic mechanical analysis (DMA). This technique involves constant amplitude oscillation of the solid to determine stress-strain properties.

\section{EXPERIMENTAL}

A DuPont 982 DMA was employed for mechanical analysis. A detailed description of the equipment has been given elsewhere $[13,14]$. The sample for the DMA was prepared by pressing 
the as-received coal powder (-100 mesh) obtained from the Argonne Premium Sample Bank with a press normally used for making FTIR sample pellets, at $15 \mathrm{kpsi}$ for 6-12 hr, which results in a sample of ca. $4 \mathrm{~mm}$ thickness with $12.8 \mathrm{~mm}$ diameter.

Slippage of the samples from the clamps inside the DMA, especially during oscillation in the high temperature range $\left(>300^{\circ} \mathrm{C}\right)$, was noted to be a main cause for irreproducibility, and special care was taken to make certain that the sample was tightly clamped. It is important to clamp the samples with approximately constant torque.

The pelletizing process used here clearly creates a solid tablet of different macroscopic mechanical properties than the original solid. The choice to work with samples prepared in this way was dictated by a desire to use the Argonne Premium Coal Samples, that are generally only available in powdered form. The gross macroscopic mechanical properties of such pellets, e.g. tensile modulus, will clearly be different from those of a sample prepared, for example, by cutting a chunk from a virgin block of coal. In fact, any samples cut directly from coal could still be subject to naturally occurring heterogeneities in the coal, and thus mechanical properties are in that case still subject to large variations, depending upon the nature of the tests. This is not important for present purposes, because our goal in the short term was not to make use of macroscopic mechanical properties. Rather, we are here only interested in changes on the molecular level that manifest themselves as changes in a particular property.

The results reported here are quantitatively inaccurate, with respect to the actual values of the tensile modulus, because the instrument was, in spite of our best attempts, prone to slip out of calibration. This means that at the moment, only trends are available, rather than exact values of the elastic constants. The instrument has been sent out for repairs, and hopefully be available soon for more quantitative work.

For present purposes of comparison, we examined the changes revealed by DMA in the context of changes earlier noted using thermal techniques, such as DSC and solvent swelling techniques. The detailed procedures for obtaining DSC and solvent swelling results have been reported elsewhere $[15,16]$. These techniques are also useful for revealing changes in the macromolecular structure, as will be noted below. 


\section{RESULTS AND DISCUSSION}

Raw DMA results are obtained as the frequency of oscillation and the damping signal. The frequency of oscillation is directly related to an elastic modulus of the sample, whereas the energy needed to maintain constant amplitude oscillation is a measure of damping within the sample [14]. Normally, the modulus is resolved into storage ( $E$ ') and loss ( $E$ ') moduli. The storage modulus corresponds to the perfectly elastic component whereas the loss modulus represents the perfectly viscous component. The dimensionless ratio of loss/storage components is defined as the damping factor, $\tan \delta\left(=E^{\prime} / E^{\prime}\right)$.

Physically, the storage modulus represents the ability of a solid to store energy elastically, whereas the loss modulus represents the ability of the systems to degrade mechanical energy to heat. The former property is desired and the latter undesirable in elastomers, such as are of interest in automobile tires. The opposite might be true in vibration isolation materials. Both properties, vary with temperature, the elastic modulus generally declining and the loss modulus often showing a peak (or several peaks) and then declining with temperature. The peaks in the loss modulus are a result of the development of mobility, in certain parts of the macromolecular structure at certain temperatures, allowing the release of accumulated strain energy as heat.

The tensile storage modulus in coal is comparable to what might be encountered in some polymer samples. We caution against placing too much emphasis on the absolute values reported here, because there were problems with instrument calibration. Instead, it is features that are clearly visible in the $\tan \delta$ or loss modulus spectra that are of significance. It is the changes in the loss modulus $\mathrm{E}^{\prime \prime}$ or the ratio, $\tan \delta=\mathrm{E}^{\prime} / \mathrm{E}^{\prime}$ that convey significant information about microscopic change in the material being tested. Both $E^{\prime \prime}$ and $\tan \delta$, for example, are used to reveal glass transitions as maxima in polymer DMA spectra. Many other transitions, in addition to a glass transition, can cause changes in $\mathrm{E}^{\prime \prime}$. Again, this parameter is a sensitive indicator of a change in the ability of molecular segments to move, relative to one another. It is this property we choose to focus on here. The loss modulus (and thus $\tan \delta$ ) will show a distinct peak at some kind of structural transition. 
In Figure 1, the lowest temperature transition (above room temperature) is seen near $60^{\circ} \mathrm{C}$, for different samples of wet, as-received Pittsburgh high volatile bituminous coal. This transition is normally not visible in DSC, because it is buried beneath the endotherm associated with water evaporation. It should be kept in mind that the values of $\tan \delta$ shown in this and subsequent figures can only be used for relative comparisons, since there was a problem with system calibration. Actual values are approximately a factor of three to five lower than shown.

The position of the peak near $60^{\circ} \mathrm{C}$ is sensitive to the presence of moisture, as is seen from Figure 2. The peak comes at lower temperature in the presence of water. This behavior is typical of the effects of a "plasticizing agent" in a polymer. The molecular motions of the coal chains are enhanced at low temperatures when water is present, and internal hydrogen bonding of the coal to itself is suppressed by the opportunity to hydrogen bond with a solvent (in this case, water). As in the case of Fig. 1, the values of E" shown in Figure 2 are strictly relative, and cannot be interpreted in absolute terms.

Considering again Fig. 1, the large peak in $\tan \delta$ above $200^{\circ} \mathrm{C}$ coincides with a transition shown both by DSC analysis of this coal and by a change in tetrahydrofuran (THF) swellability (see Figure 3). For convenience, Figure 3a shows a crossplot of the data for undried, as-received Pittsburgh No. 8 obtained in both DSC and DMA. The transition revealed by the difference DSC is dramatically revealed by the DMA. The JMA analyses were not carried out to as high a temperature as the DSC analyses because of the failure of samples at higher temperatures. The transition near $275^{\circ} \mathrm{C}$ involves an irreversible relaxation of the coal structure [16,17].

Figure 4 illustrates the behavior of Upper Freeport medium volatile bituminous coal in DMA. The tensile storage modulus exhibits a continuous decline with temperature, except for two regions of more rapid decline in modulus. The loss modulus, and thus $\tan \delta$, both suggest that there is a low temperature event, again probably associated with moisture in the coal, at below $100^{\circ} \mathrm{C}$. The main relaxation of structure starts to occur from $240^{\circ} \mathrm{C}$, as observed in both tensile storage modulus and $\tan \delta$. This observation augments the DSC and solvent swelling results (see Figure 5) for the same coal, in that Upper Freeport coal showed the characteristics of a relaxing coal structure, such as increased swellability in solvents and an endothermic relaxation peak. In 
the DSC, the coal exhibited a distinct endothermic peak centered around $350^{\circ} \mathrm{C}$ which was started from around $310^{\circ} \mathrm{C}$, whereas solvent swellability increased significantly above $250^{\circ} \mathrm{C}$. It is thus confirmed by DMA that solvent swelling is a more sensitive indicator of irreversible structural relaxation than is DSC.

Finally, Fig. 6 shows a comparison of difference DSC results and DMA results for undried Powhatan No. 5 HVB coal. There is an interesting event that reveals itself as an irreversible endotherm at near $300^{\circ} \mathrm{C}$ in the DSC. There is no clean signature in the DMA that coincides with this endotherm; rather there is a general increase in $\tan \delta$ in the same temperature.

It should be noted that there are not "clean" DMA peaks observable in the high temperature range of any of the DMA spectra shown. This is because the measurements could never be reliably carried out to high enough temperatures to fully resolve the peak. The samples always failed when temperatures approached those necessary for pyrolysis $\left(>325^{\circ} \mathrm{C}\right)$. Thus the technique is limited in applicability to near ambient conditions. For purposes of obtaining elastic constants, this is not a significant drawback, since these measurements will generally be performed near room temperature.

\section{CONCLUSIONS}

Three bituminous coals were subjected to DMA analysis and the results were compared to DSC and solvent swelling results. Results from different techniques appear to point to the same basic conclusions with regard to transitions, although there are subtle differences between the results of different techniques. The transition related to coal moisture is more visible in DMA and solvent swelling techniques than in DSC analysis. DMA confirms that solvent swellability is a more sensitive index of macromolecular changes than is DSC. The importance of observations made by several different techniques in order to discern transitions accurately has been noted. This study confirms the usefulness of applying different techniques simultaneously for this purpose.

We noticed that good reproducibility in DMA depends upon the reproducibility of forming

pellets, in our case. A significant experimental problem involved small cracks generated while the pellet was released from the press. We are further pursuing these problems in order to obtain a 
better description of transitions and elastic properties of coal.

\section{REFERENCES}

1. Van Krevelen, D.W. In Coal; Elsevier, New York, 1981; Chapter XX.

2. Hiorns, F.J. Fuel 1953, 32, 113.

3. Terry, N.B. Fuel 1958, 37, 309.

4. Morgans, W.T.A.; Terry, N.B. Fuel 1958, 37, 201.

5. Hall, P.J.; Marsh, H.; Thermas, K.M., unpublished results, University of Newcastle-UponTyne, 1988.

6. Brenner, D. Prepr. Pap.-Am. Chem. Soc., Div. Fuel Chem.1986, 31(1), 17.

7. Weller, M.; Wert, C. Fuel 1984, 63, 891.

8. Wert, C.A.; Weller, M.; Caulfield, D. J. Appl. Phys. 1984, 56(9), 2453.

9. Wert, C.A.; Weller, M. J. Appl. Phys. 1982, 53(10), 6505.

10. Weller, M; Wert, C. Int. Conf. on Coal Science 1987, 65.

11. Toda, Y.; Toyoda, S. Fuel 1972, 51, 199.

12. Debelak, K.A.; Schrodt, J.T. Fuel 1979, 58, 732.

13. Wetton, R.E. Polymer Testing 1984, 4, 117.

14. Gill, P.S.; Lear, J.D.; Leckenby, J.N. Polymer Testing 1984, 4, 131.

15. Yun, Y.; Suuberg, E.M. Prepr. Pap.-Am. Chem. Soc., Div. Fuel Chem. 1992, 37(2), 856.

16. Yun, Y.; Suuberg, E.M. Fuel 1993, in press.

17. Yun, Y.; Suuberg, E.M. Energy \& Fuels 1992, 6, 328. 


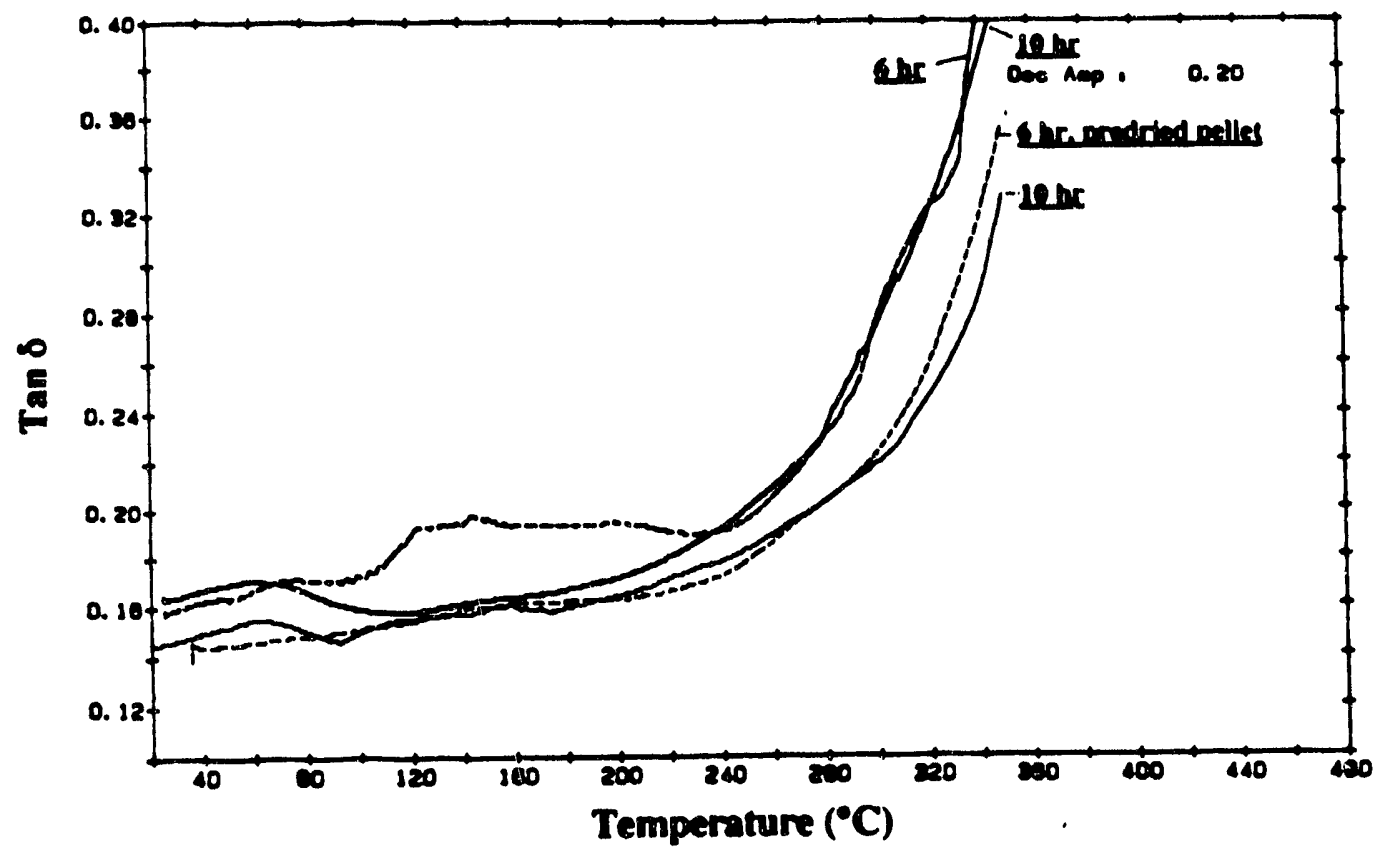

Figure 1. Tan $\delta$ DMA spectra obtained from $4^{\circ} \mathrm{C} / \mathrm{min}$ scans of as-received and predried (up to $200^{\circ} \mathrm{C}$ at $4^{\circ} \mathrm{C} / \mathrm{min}$ ) Pittsburgh No. 8 coal pellet samples. Pellets were made from -100 mesh powder after pressed at $15 \mathrm{kpsi}$ for the duration as specified in the figure.

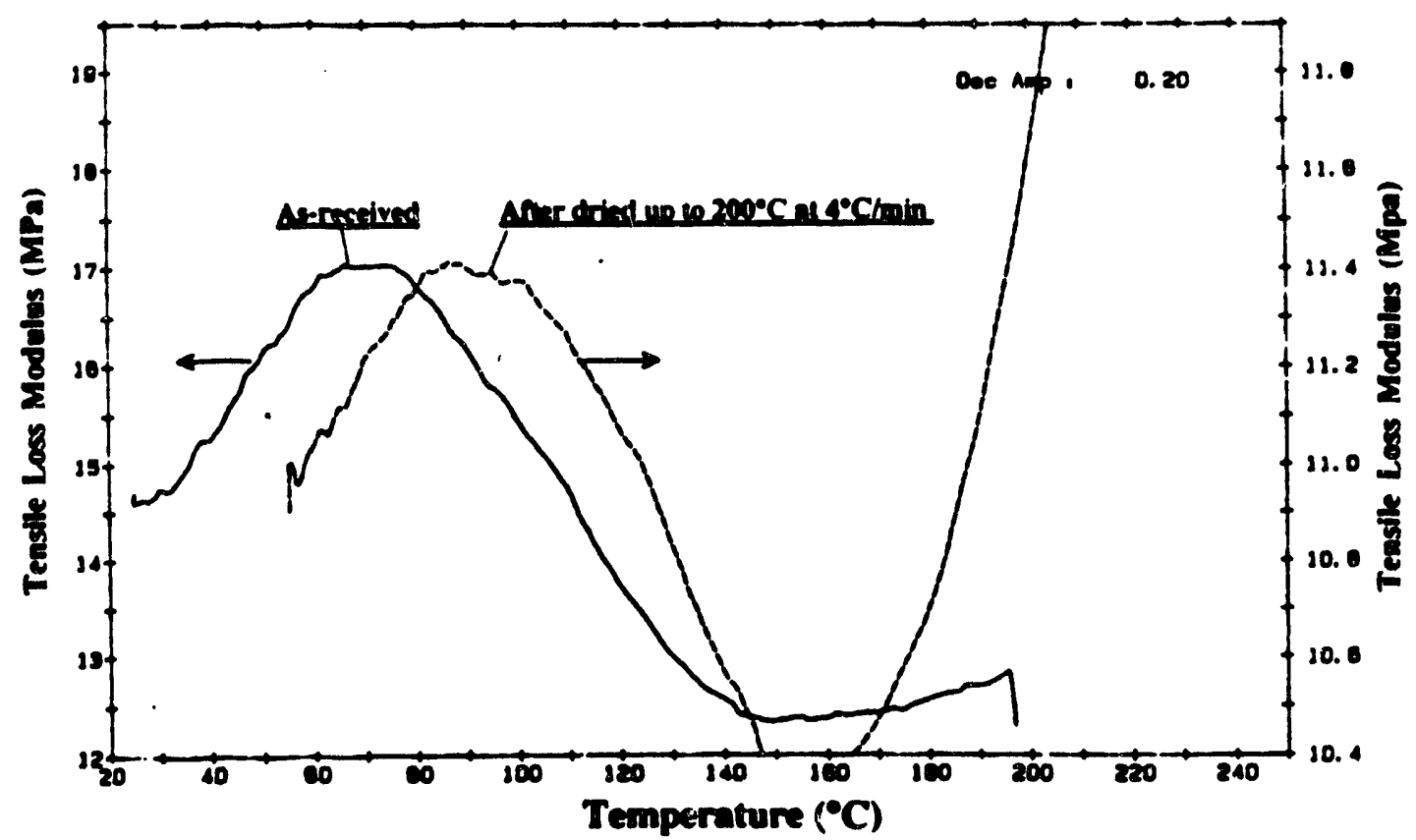

Figure 2. Effect of sample drying on tensile loss modulus obtained at $4^{\circ} \mathrm{C} / \mathrm{min}$ for Pittsburgh No. 8 coal. Sample pellets were made from -100 mesh powder pressed at $15 \mathrm{kpsi}$ for $10 \mathrm{hr}$. 


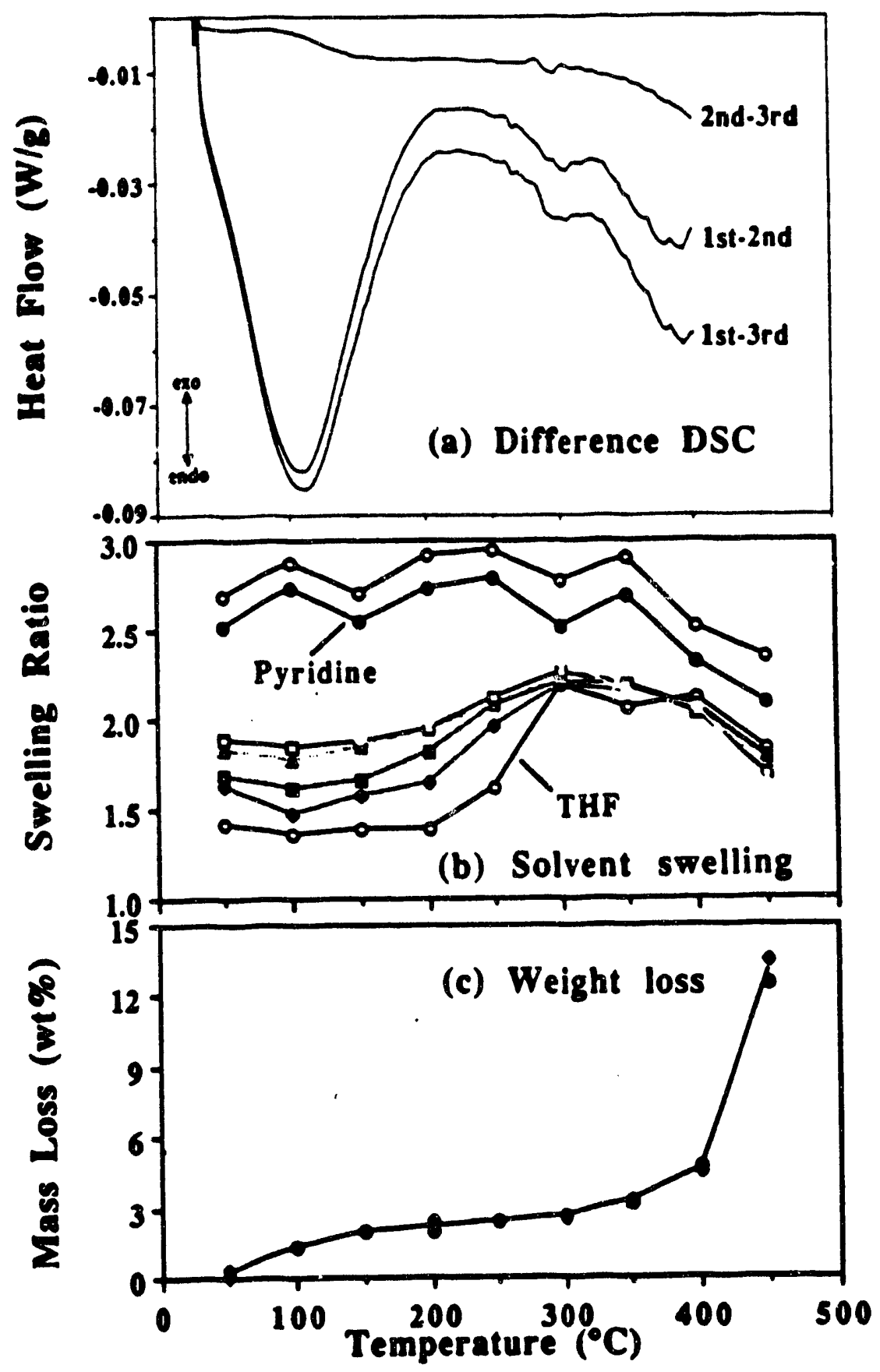

Figure 3. Difference DSC thermograms as well as profiles of solvent swelling ratio and weight loss obtained at $8^{\circ} \mathrm{C} / \mathrm{min}$ from -100 mesh Pittsburgh No. 8 coal powder (swelling time:Q, $5 \mathrm{hr} ; 0$, 1 day; $\bullet, 2$ days; $₫, 4$ days; 0,5 days; $\bullet, 6$ days). 


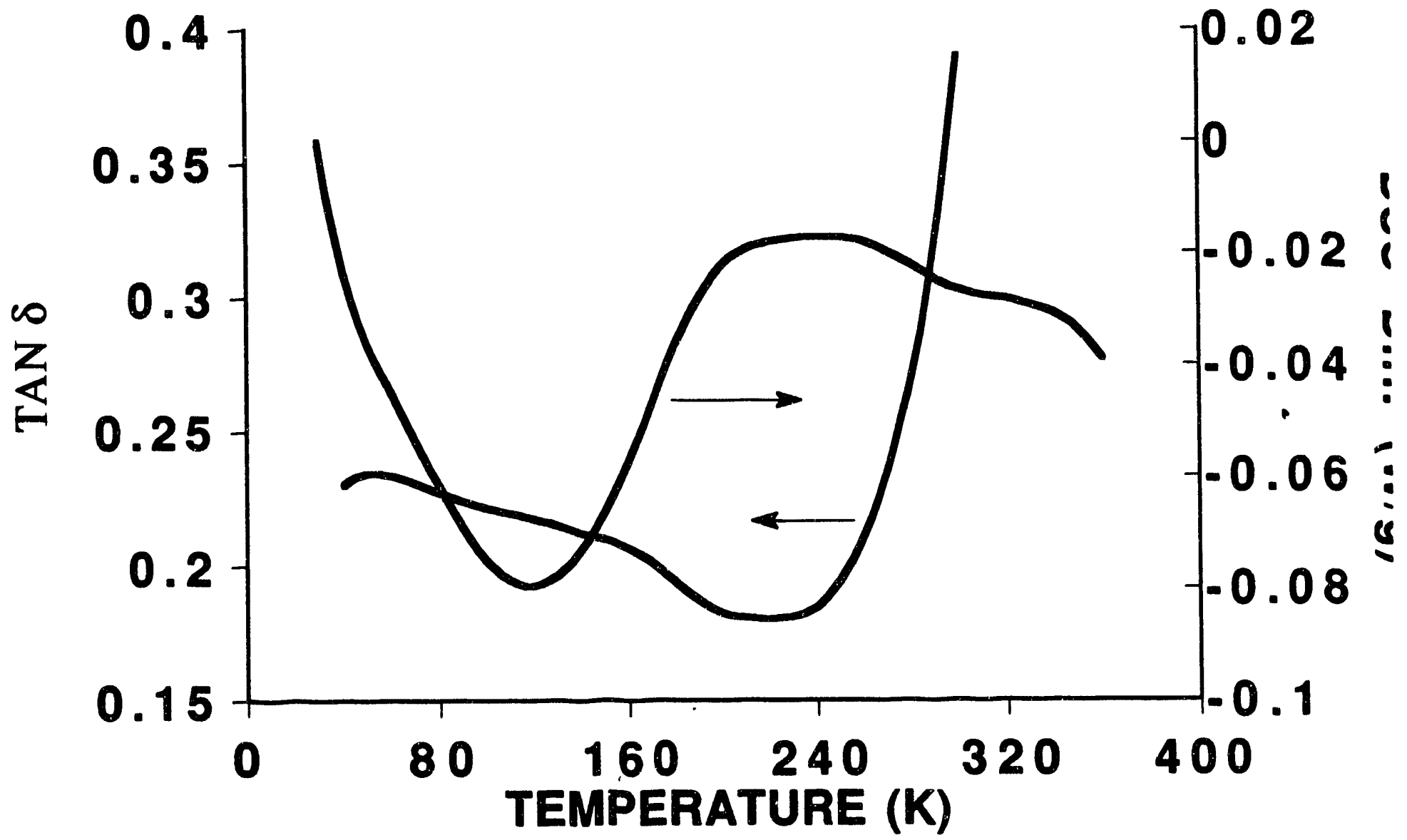

Figure 3a. Details of the DSC difference spectrum and the DMA spectrum for Pittsburgh No. 8 coal. The DSC curve was obtained at $8 \mathrm{~K} / \mathrm{min}$ and the DMA at $4 \mathrm{~K} / \mathrm{min}$. 


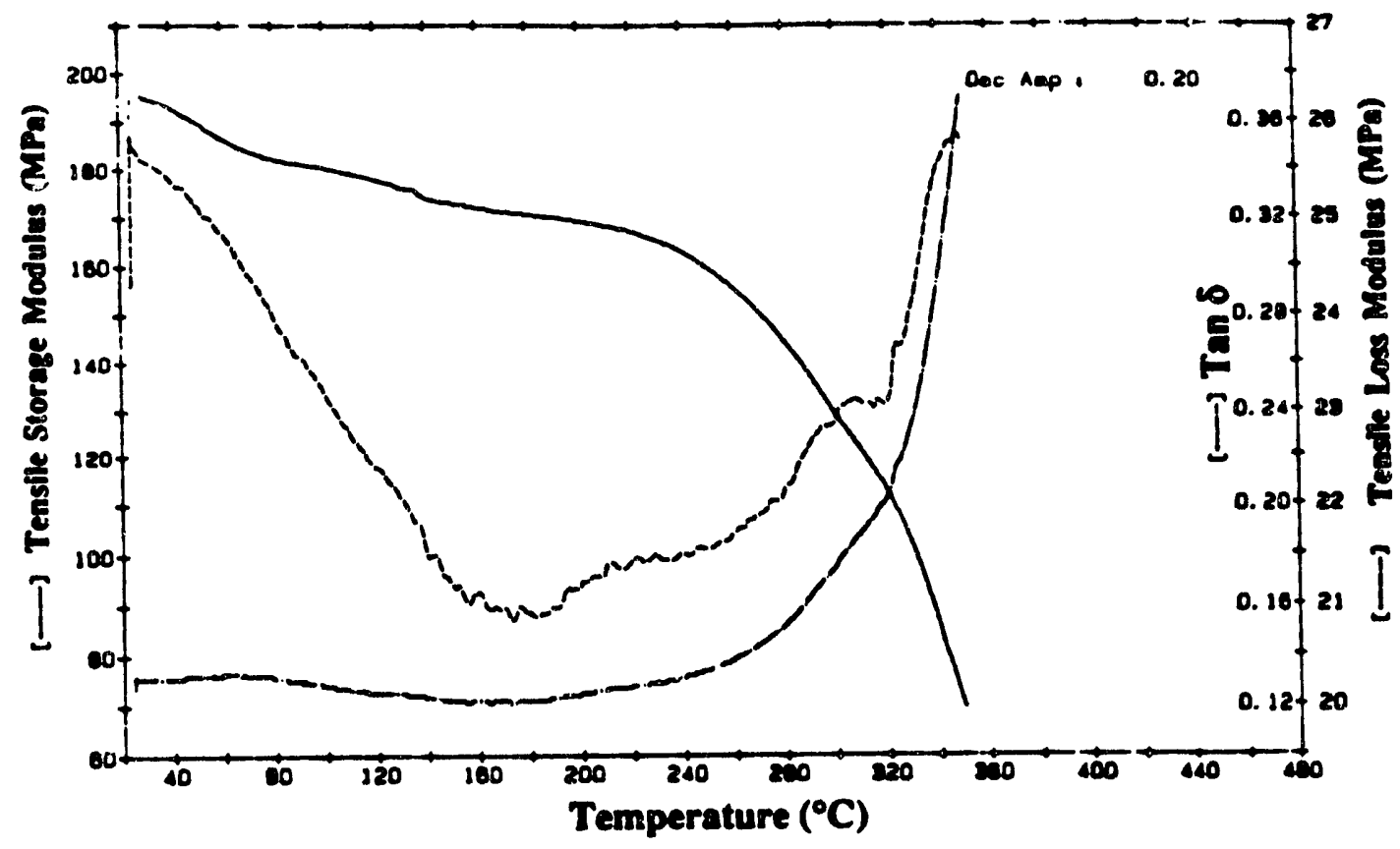

Figure 4. DMA scan of the Upper Freeport medium volatile bituminous coal obtained at $4^{\circ} \mathrm{C} / \mathrm{min}$. Sample pellet was made from -100 mesh powder pressed at $15 \mathrm{kpsi}$ for $12 \mathrm{hr}$.
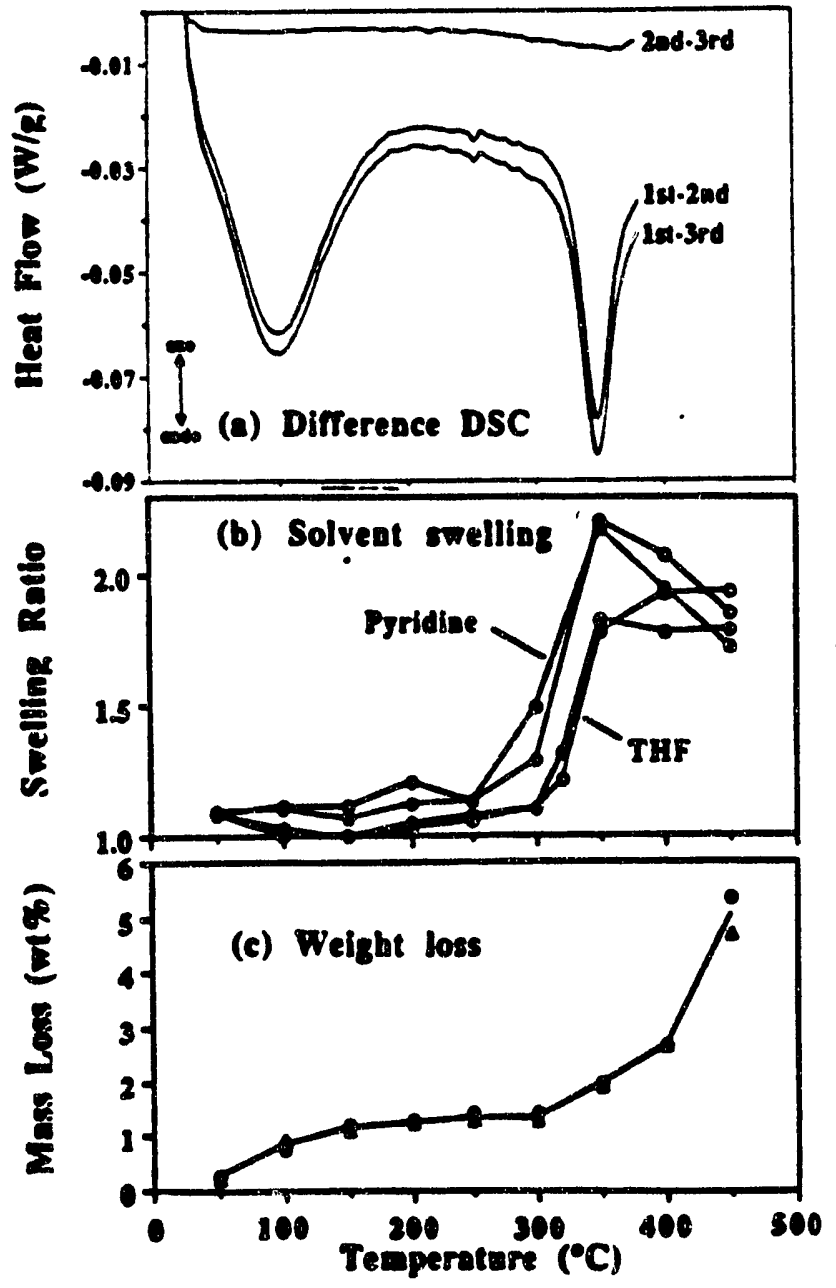

Figure 5. Difference DSC thermograms as well as profiles of solvent swelling ratio and weight loss obtained at $8^{\circ} \mathrm{C} / \mathrm{min}$ from -100 mesh Upper Freeport coal powder (swelling time:0,5 hr,a 7 days). 


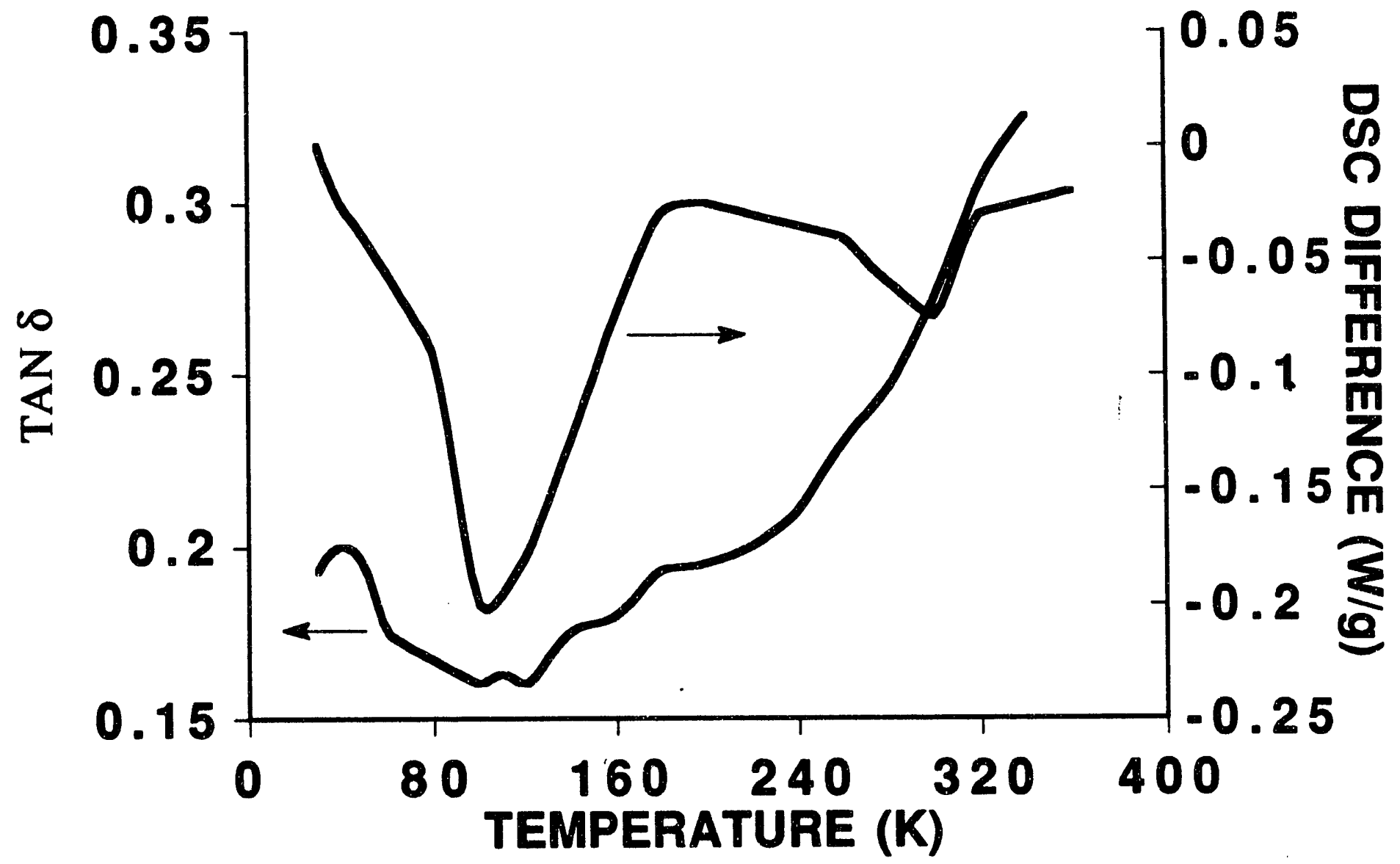

Figure 6. Difference DSC spectrum and DMA spectrum for Pocahontas No. 5 HVB coal. The difference DSC spectra were obtained at $8 \mathrm{~K} / \mathrm{min}$, and the DMA at $4 \mathrm{~K} / \mathrm{min}$. 

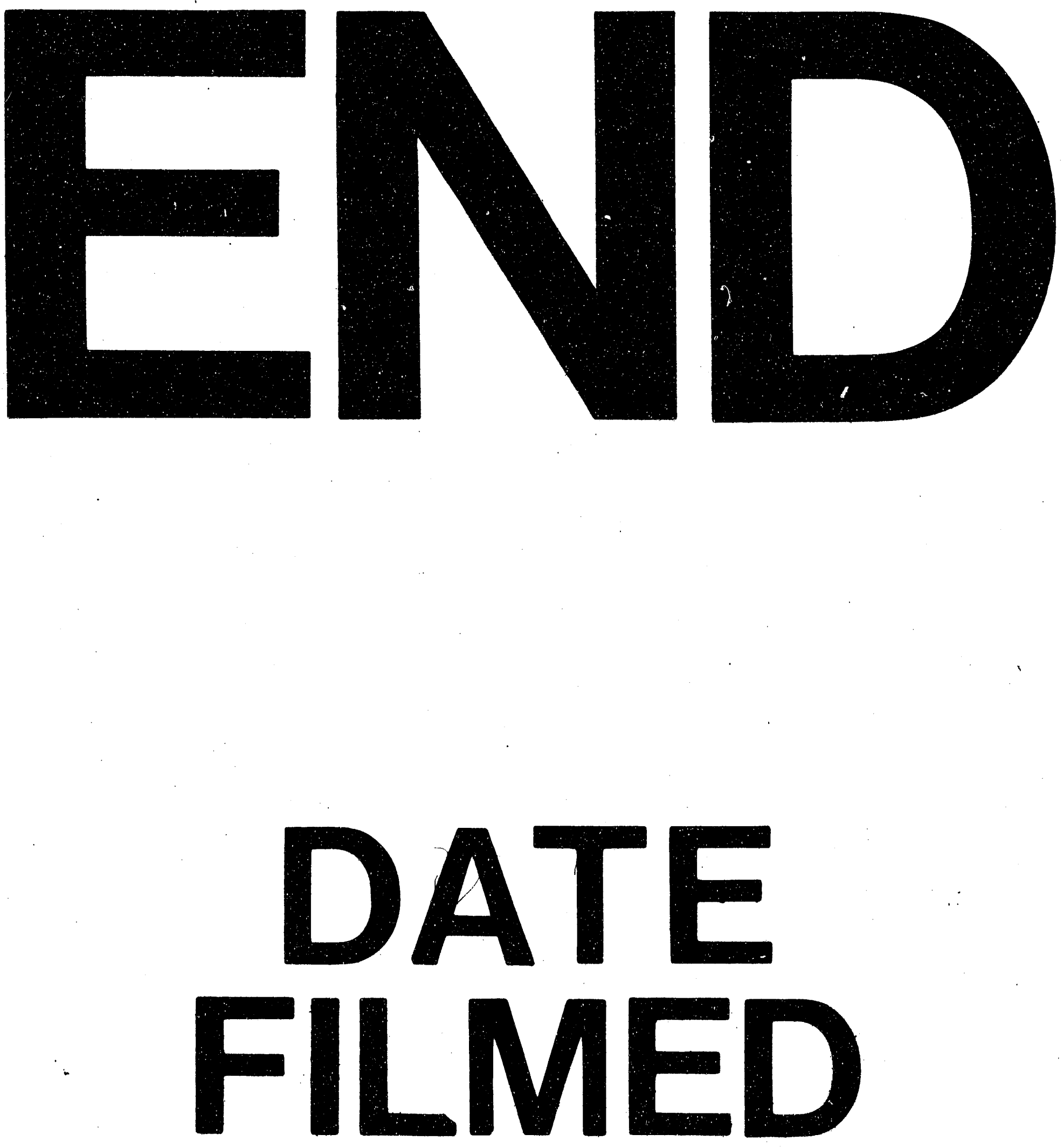

I

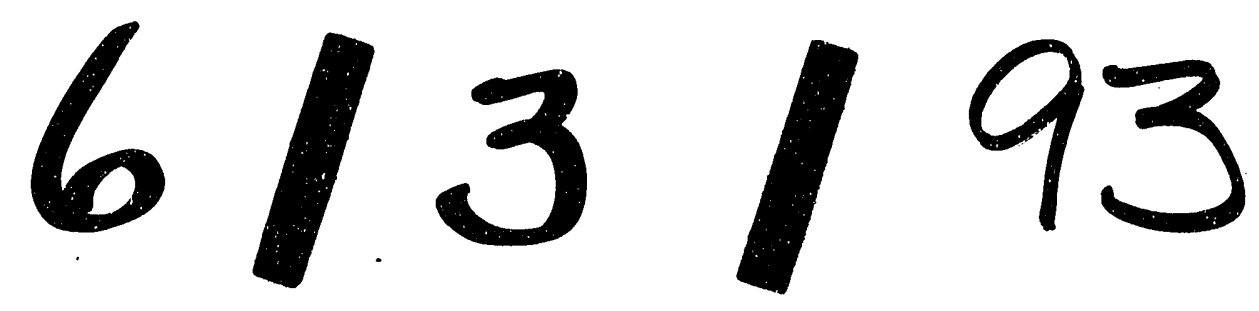


